\title{
Kinetic simulation of an extreme ultraviolet radiation driven plasma near a multilayer mirror
}

\section{Citation for published version (APA):}

Velden, van der, M. H. L., Brok, W. J. M., Mullen, van der, J. J. A. M., \& Banine, V. Y. (2006). Kinetic simulation of an extreme ultraviolet radiation driven plasma near a multilayer mirror. Journal of Applied Physics, 100(7), 073303-1/7. [073303]. https://doi.org/10.1063/1.2356085

DOI:

$10.1063 / 1.2356085$

Document status and date:

Published: 01/01/2006

\section{Document Version:}

Publisher's PDF, also known as Version of Record (includes final page, issue and volume numbers)

\section{Please check the document version of this publication:}

- A submitted manuscript is the version of the article upon submission and before peer-review. There can be important differences between the submitted version and the official published version of record. People interested in the research are advised to contact the author for the final version of the publication, or visit the $\mathrm{DOI}$ to the publisher's website.

- The final author version and the galley proof are versions of the publication after peer review.

- The final published version features the final layout of the paper including the volume, issue and page numbers.

Link to publication

\section{General rights}

Copyright and moral rights for the publications made accessible in the public portal are retained by the authors and/or other copyright owners and it is a condition of accessing publications that users recognise and abide by the legal requirements associated with these rights.

- Users may download and print one copy of any publication from the public portal for the purpose of private study or research.

- You may not further distribute the material or use it for any profit-making activity or commercial gain

- You may freely distribute the URL identifying the publication in the public portal.

If the publication is distributed under the terms of Article 25fa of the Dutch Copyright Act, indicated by the "Taverne" license above, please follow below link for the End User Agreement:

www.tue.nl/taverne

Take down policy

If you believe that this document breaches copyright please contact us at:

openaccess@tue.nl

providing details and we will investigate your claim. 


\title{
Kinetic simulation of an extreme ultraviolet radiation driven plasma near a multilayer mirror
}

\author{
M. H. L. van der Velden, ${ }^{a)}$ W. J. M. Brok, and J. J. A. M. van der Mullen \\ Department of Applied Physics, Eindhoven University of Technology, P.O. Box 513, 5600 MB Eindhoven, \\ The Netherlands \\ V. Banine \\ ASML Netherlands B.V., De Run 6501, 5504 DR Veldhoven, The Netherlands
}

(Received 28 March 2006; accepted 27 June 2006; published online 6 October 2006)

\begin{abstract}
Future generation lithography tools will use extreme ultraviolet radiation to enable the printing of sub-50 nanometer features on silicon wafers. The extreme ultraviolet radiation, coming from a pulsed discharge, photoionizes the low pressure background gas in the tool. A weakly ionized plasma is formed, which will be in contact with the optical components of the lithography device. In the plasma sheath region ions will be accelerated towards the surfaces of multilayer mirrors. A self-consistent kinetic particle-in-cell model has been applied to describe a radiation driven plasma. The simulations predict the plasma parameters and notably the energy at which ions impact on the plasma boundaries. We have studied the influence of photoelectron emission from the mirror on the sheath dynamics and on the ion impact energy. Furthermore, the ion impact energy distribution has been convoluted with the formula of Yamamura and Tawara [At. Data Nucl. Data Tables 62, 149 (1996)] for the sputter yield to obtain the rate of physical sputtering. The model predicts that the sputter rate is dominated by the presence of doubly ionized argon ions. (C) 2006 American Institute of Physics. [DOI: 10.1063/1.2356085]
\end{abstract}

\section{INTRODUCTION}

Over the last two decades lithography equipment, used in the chip-making industry, has seen a continuous shift towards shorter operating wavelengths in order to achieve shrinking device sizes and faster chip performance. Next generation lithography tools will use extreme ultraviolet (EUV) radiation with wavelength of $13.5 \mathrm{~nm} .{ }^{1,2}$ As EUV radiation is strongly absorbed in virtually all materials, refractive optical elements, such as lenses cannot be used and instead multilayer coated mirrors ${ }^{3}$ are required for imaging.

Furthermore, to decrease absorption losses, the optical path is enclosed in a vacuum system. However, the residual argon background gas at a pressure of 0.1-1 $\mathrm{Pa}$ will be partially photoionized by the EUV radiation, which will generate a weakly ionized plasma in the tool.

Like in any bound plasma, a plasma sheath will develop in which the ions will be accelerated towards the plasma boundaries such as multilayer mirrors. Provided the potential drop across the plasma sheath is large enough, the ions will gain sufficient kinetic energy to cause damage to the multilayer mirrors through physical sputtering. Our aim is to model the plasma sheath region to calculate the flux and energy of the ions impacting on the mirrors.

The plasma description is made more arduous by the transient nature of the EUV sources. A hollow cathode discharge ${ }^{4}$ is employed to generate EUV pulses with a duration of approximately $100 \mathrm{~ns}$ at a repetition frequency of typically $1 \mathrm{kHz}$.

Because of the low plasma density $\left(n_{e} \approx 10^{15} \mathrm{~m}^{-3}\right)$ and

${ }^{a)}$ Electronic mail: m.h.l.v.d.velden@tue.nl the strong time dependence, the plasma will be far from equilibrium. The low pressure implies that the plasma is in the nonlocal regime and a kinetic model is required for an accurate description. Hereto, we apply a particle-in-cell (PIC) model. ${ }^{5}$ The main advantage of such a model is that it enables the self-consistent calculation of the fields and the energy and velocity distributions without the need for equilibrium assumptions. ${ }^{6}$ PIC models have the disadvantage that they are computationally demanding. ${ }^{7}$ Collisions between charged species and background neutrals can be represented statistically by combining PIC methods with Monte Carlo collisions (MCCs). ${ }^{8,9}$

In our earlier work ${ }^{10}$ a PIC-MCC model was presented that had been applied to describe the development of the plasma sheath and to calculate the energy at which the ions impact on the mirror. It was found that the impact energies of the ions are in the near-threshold sputter regime, ${ }^{11}$ where the sputter yield (i.e., the number of atoms removed per incoming ion) strongly depends on the ion impact energy.

In this paper we aim to study the effect of photoelectron emission from the mirror surface on the energies of the ions impacting on the mirror. The electrons that are emitted due to the photoelectric effect when the EUV radiation strikes the mirror will influence the sheath dynamics.

Furthermore, we have added the double photoionization process to the model. Doubly ionized atoms will gain twice the amount of energy in the plasma sheath compared to singly ionized atoms and will therefore substantially contribute to the sputter rate.

Details of the PIC-MCC model can be found in Ref. 10 and we will only give a short description here. 


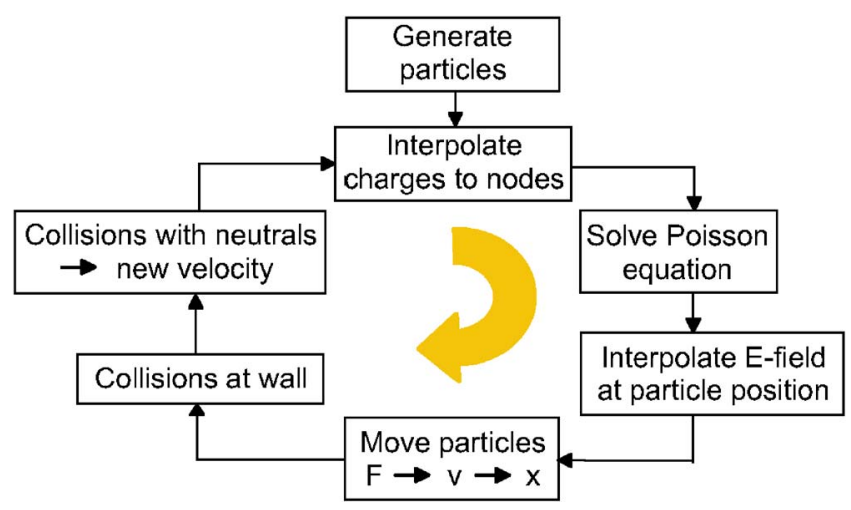

FIG. 1. (Color online) Particle-in-cell scheme.

\section{MODEL DESCRIPTION}

The PIC-MCC code is one dimensional in configuration and three dimensional in velocity space. Charged species are computationally represented by "superparticles." Each superparticle corresponds to typically $10^{9}$ real particles. A computational grid divides the plasma into a number of cells. Each cell must contain a sufficient number of particles $(>100)$ to ensure proper statistics.

The general scheme of the explicit PIC model is shown in Fig. 1. For each time step $\Delta t$, (1) the particle charges are assigned to the nodes of (2) the computational grid by a linear weighing, ${ }^{12}$ the Poisson equation is solved on the nodes to obtain the electric field, (3) a linear weighing is applied to find the electric field at the position of each superparticle, (4) the Newtonian equations of motions are applied to advance the positions and velocities of the superparticles using a second order leapfrog scheme, (5) the particles that have moved beyond the boundaries of the computational grid are removed from the simulation, and (6) the MCC routine checks if a particle has collided with the background gas and adjusts the velocity accordingly. Additional superparticles are created in the case of an ionizing collision.

In the model, only collisions between charged particles and neutrals are taken into account. Collisions between charged particles can be neglected due to the low plasma density. A collision is treated here as an instantaneous process that changes the particle's velocity in both magnitude and direction. We use the "null collision" technique ${ }^{13}$ to determine the free-flight time of each particle between collisions. Because of the low ionization degree of the plasma, we can assume a homogeneous neutral density.

When a collision takes place, it is stochastically determined which type of collision occurs based on the relative collision frequencies of the relevant processes. The collision frequency of each process is proportional to the collisional cross section for that collision type and to the velocity of the particle. The following binary collision types are taken into account:

(1) elastic electron-neutral collisions

$e^{-}+\mathrm{Ar} \rightarrow e^{-}+\mathrm{Ar}$,

(2) inelastic electron-neutral collisions

$e^{-}+\mathrm{Ar} \rightarrow e^{-}+\mathrm{Ar}^{*}$,

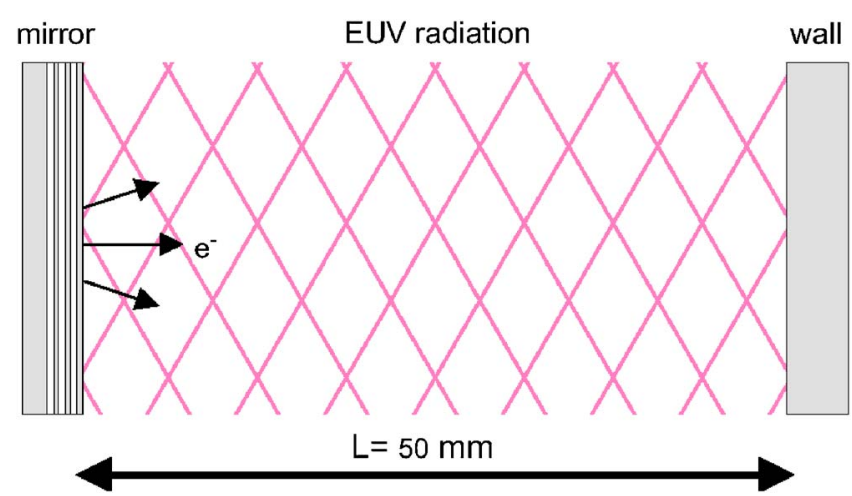

FIG. 2. (Color online) Schematic of the geometry used in calculations. The EUV radiation is partially reflected by the mirror. The EUV radiation between the mirror on the left and a plain wall on the right is assumed to be monochromatic and homogeneous in intensity. Photoelectrons are only emitted from the surface of the mirror.

(3) electron-impact ionization collisions

$e^{-}+\mathrm{Ar} \rightarrow 2 e^{-}+\mathrm{Ar}^{+}$

(4) elastic ion-neutral collisions

$\mathrm{Ar}^{+}+\mathrm{Ar} \rightarrow \mathrm{Ar}^{+}+\mathrm{Ar}$

(5) charge-exchange collisions

$\mathrm{Ar}^{+}+\mathrm{Ar} \rightarrow \mathrm{Ar}+\mathrm{Ar}^{+}$.

Cross sections for these processes are the same as the ones used by Phelps and Petrovic. ${ }^{14}$

First, upon collision, the velocities of both particles are transformed to the center-of-mass frame. Second, the scattering angle is determined stochastically based on the angular cross section at that energy. Third, the velocities of the particles are adjusted accordingly, and fourthly, the particle velocities are transformed back to the laboratory frame.

\section{A. Plasma generation}

In our simulations the geometry shown in Fig. 2 is used. The EUV driven plasma is bounded by a multilayer mirror on one side and a metal wall on the other side. The mirror is assumed to have a ruthenium surface because in EUV lithography this metal is often applied as a capping layer (thickness $\approx 1.5 \mathrm{~nm}$ ) to provide a barrier against oxidation of the underlying $\mathrm{Mo} / \mathrm{Si}$ stacks. ${ }^{15}$ The EUV radiation is partially reflected by the mirror with a reflection coefficient of $R_{\mathrm{ml}}$ $=68 \%$, which is typical for Mo/Si multilayer mirrors. ${ }^{1,16}$ The remaining $32 \%$ of the radiation is absorbed and is converted primarily to heat. In the model, the interaction between the EUV radiation and the wall is not taken into account. Both mirror and wall are assumed to be grounded.

Because the bandwidth of the radiation used in EUV lithography is very narrow (2\%), we can make the approximation that the EUV radiation is monochromatic. The EUV radiation is assumed to have a uniform intensity with a temporal distribution $G(t)$ that is modeled with a cutoff Gaussian with a total duration of $2 \tau=100 \mathrm{~ns}$

$$
G(t)=\frac{\alpha I_{p}}{\tau} \exp \left[-\frac{(t-\tau)^{2}}{2 \tau^{2}}\right] \text { for } 0<t<2 \tau,
$$

with $\alpha \approx 0.584$ a numerical constant to normalize $G(t)$ to the pulse-averaged EUV irradiance $I_{p}$. 
Charged particles are generated in two ways: (1) electron-ion pairs as a result of photoionization of the argon background gas and (2) emission of electrons from the mirror surface as a result of the photoelectric effect.

\section{Photoionization}

The EUV photon energy of $h \nu=92 \mathrm{eV}$ exceeds the argon photoionization threshold of $E_{s}=15.8 \mathrm{eV}$. In case of a photoionization event, an electron-ion pair is generated in the volume. As the momentum carried by the photon is negligible, the sum of the momenta of the created electron and ion must be approximately zero. This implies that most of the excess energy $T_{e}=h \nu-E_{s}$ will be transferred to the electron, because the $\mathrm{Ar}^{+}$ion is much heavier than the electron. In the model it is assumed that the ion kinetic energy is equal to the thermal energy of the neutral prior to ionization. The ion, created in the photoionization process, receives an amount of kinetic energy sampled from a Maxwellian energy distribution at room temperature $(T=300 \mathrm{~K})$.

The threshold for double photoionization of argon is $E_{d}=43.4 \mathrm{eV}$. This means that besides singly ionized, doubly ionized argon ions will also be formed. For every double photoionization event one $\mathrm{Ar}^{2+}$ ion and two electrons are created. It is assumed that the $\mathrm{Ar}^{2+}$ ion receives the kinetic energy of room temperature neutral argon, whereas the excess energy $T_{e}=h \nu-E_{d}$ is randomly distributed over the two electrons, i.e., the first electron receives an amount of energy $E_{1}=R\left(h \nu-E_{d}\right)$ and the second electron $E_{2}=(1-R)\left(h \nu-E_{d}\right)$, with $R \in[0,1)$ a random number.

The total number $N_{\text {pi }}$ of single photoionization events per EUV pulse per $\mathrm{m}^{3}$ is equal to

$$
N_{\mathrm{ph}}=\frac{2 \tau I_{p}}{h \nu L}\left[1-\exp \left(-n_{\mathrm{Ar}} \sigma_{s} L\right)\right]\left[1+R_{\mathrm{ml}} \exp \left(-n_{\mathrm{Ar}} \sigma_{s} L\right)\right],
$$

where the factor $2 \tau$ arises from the integration of $I_{p}$ over the pulse duration, with $L$ the distance between the multilayer mirror and the wall, $n_{\mathrm{Ar}}$ the argon density, and $\sigma_{s}=1.4$ $\times 10^{-22} \mathrm{~m}^{2}$ the single photoionization cross section at $92 \mathrm{eV} .{ }^{17}$ Equation (2) takes into account the partial reflection of the EUV beam from the mirror with reflectivity coefficient $R_{\mathrm{ml}}$. Because $n_{\mathrm{Ar}} \sigma_{s} L \ll 1$, the plasma is optically thin for EUV radiation, and we can make the following approximation:

$$
N_{\mathrm{ph}} \approx \frac{2 \tau I_{p}}{h \nu}\left(1+R_{\mathrm{ml}}\right) n_{\mathrm{Ar}} \sigma_{s}
$$

The same formula can be applied to calculate the total number of double photoionization events by replacing $\sigma_{s}$ with the double photoionization cross section $\sigma_{d}=2.1 \times 10^{-23} \mathrm{~m}^{2}{ }^{18}$

\section{Photoelectric effect}

The EUV radiation is partially absorbed in the multilayer mirror leading to the emission of photoelectrons from the surface (photoelectric effect). In case photon absorption takes place close to the surface, the generated primary electrons can directly escape the solid with a kinetic energy equal to the photon energy reduced by the work function or surface
TABLE I. Simulation parameters.

\begin{tabular}{lcc}
\hline \hline Background density & $n_{\mathrm{Ar}}$ & $1.2 \times 10^{20} \mathrm{~m}^{3}$ \\
EUV irradiance & $I_{p}$ & $6 \times 10^{6} \mathrm{~W} \mathrm{~m}^{-2}$ \\
Time step & $\Delta t$ & $1 \times 10^{-12} \mathrm{~s}$ \\
Particle weight & $\mathrm{PW}$ & $10^{9}$ \\
Number of cells & $N$ & 300 \\
Length & $L$ & $5 \times 10^{-2} \mathrm{~m}$ \\
\hline \hline
\end{tabular}

binding energy of the solid. However, as the absorption length for EUV radiation is much larger than the mean free path for electron-electron collisions in the solid, in most cases the primary electrons will undergo many scattering events before reaching the surface. This leads to the emission of low-energy secondary electrons from the solid. For photon energies above $100 \mathrm{eV}$ the shape of the energy distribution of secondary electrons emitted from the surface is essentially independent ${ }^{19}$ of the photon energy and is given by

$$
S\left(E_{e}, W\right)=\frac{6 W^{2} E_{e}}{\left(E_{e}+W\right)^{4}},
$$

where $W$ is the work function or surface binding energy. We can sample the electron energy from this distribution according to

$$
E_{e}=W\left[\sqrt{\frac{4}{1-R}} \cos \left(\frac{\pi-\arccos \sqrt{(1-R)}}{3}\right)-1\right],
$$

with $R \in[0,1)$ a random number. The emitted electrons are assumed to have a $\cos (\theta)$ distribution around the surface normal, which is a good approximation as long as the electrons that are generated inside the solid by the photoionization process are distributed isotropically.

\section{RESULTS AND DISCUSSION}

The simulation parameters are listed in Table I. The chosen background pressure and pulse-averaged EUV irradiance are typical for our laboratory EUV setup. The length of the computational domain is divided into $N=300$ cells, so that the cell size is smaller than the Debye screening length $\lambda_{D}$, which is the characteristic length scale for charge separation in a plasma. For our application $\lambda_{D} \approx 5 \times 10^{-4} \mathrm{~m}$. Each species is simulated with $10^{5}$ superparticles to ensure proper statistics. The time step of the PIC loop must be small compared to the time it takes for a fast electron to travel across a cell, which for a $100 \mathrm{eV}$ electron and for our cell size corresponds to $\approx 30 \mathrm{ps}$. This is two orders of magnitude smaller than the fastest time scale of the plasma, which is given by the inverse of the plasma electron frequency.

We investigate the following three cases to study the effect of the photoelectrons on the ion sputtering:

(a) No photoeffect.

(b) Primary photoelectrons only. The photoelectrons each have kinetic energy $h \nu-W$.

(c) Secondary photoelectrons only. A photoelectron has a kinetic energy sampled from the energy distribution of Eq. (4), which is shown in Fig. 3. 


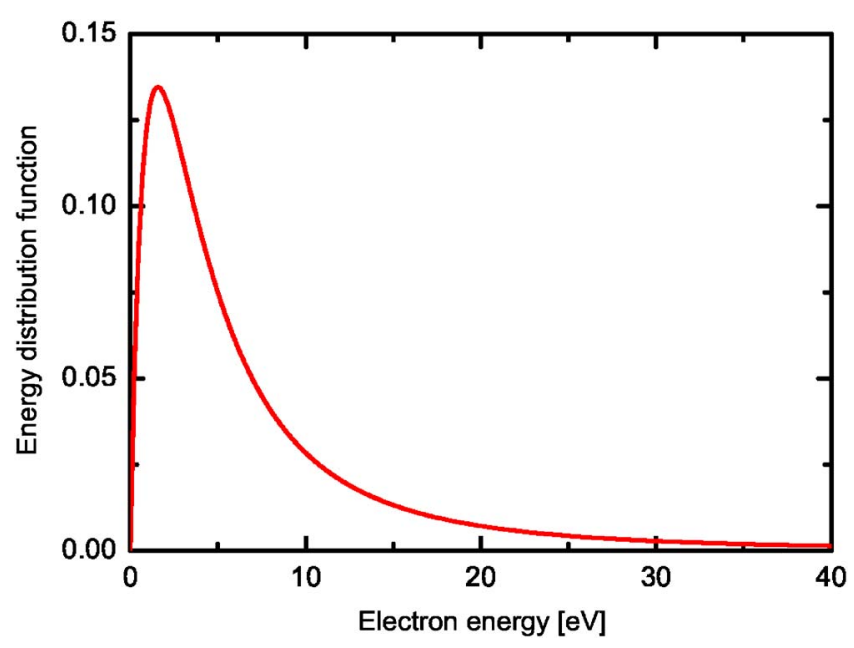

FIG. 3. (Color online) Energy distribution of secondary photoelectrons emitted from the mirror [Eq. (4)].

In reality, about $50 \%-90 \%$ of the emitted photoelectrons can be expected to be low-energy secondary photoelectrons. ${ }^{19}$

\section{A. Plasma sheath}

The formation of the plasma sheath near the mirror can be seen in Fig. 4, where the plasma density profile is shown at the start of the EUV pulse $(t=10 \mathrm{~ns})$, at the maximum EUV intensity $(t=50 \mathrm{~ns})$, and after the EUV pulse $(t$ $=500 \mathrm{~ns}$ ). Figure 4(a) shows the case of no photoeffect. The depicted effective ion density $n_{i}^{*}=\rho^{+} / e$ is equal to the charge density of all positive ions (both singly and doubly charged) scaled by the elementary charge. Near the mirror the ion density exceeds the electron density as the highly mobile electrons are lost at the wall leaving the more inert ions behind. At the end of the EUV pulse, most electrons still have sufficient kinetic energy to cause electron-impact ionization processes. Therefore, the plasma density reaches a maximum when the EUV pulse has already ended. It was found that the maximum of $n_{e}=3 \times 10^{15} \mathrm{~m}^{-3}$ is attained $500 \mathrm{~ns}$ after the start of the $100 \mathrm{~ns}$ long EUV pulse. After that the plasma density starts to decay on a time scale that is long compared to the pulse duration, but short compared to the time between EUV pulses.

The photoemission from the mirror changes the dynamics of the sheath buildup [Figs. 4(b) and 4(c)]. At the start of the plasma formation ( $t=10 \mathrm{~ns})$ the electron density exceeds the ion density near the mirror due to the emission of photoelectrons. In the case of secondary photoelectrons, the negative space charge is much more concentrated near the mirror than in the case of the more energetic primary photoelectrons, which penetrate further into the plasma in the same amount of time. Somewhat later $(t=50 \mathrm{~ns})$ the volume effect becomes dominant as the electrons from the gas phase have had time to escape to the mirror surface.

Figure 5 shows the cell-averaged energy of the plasma electrons as a function of position. Initially, the electron energy of the electrons is close to $h \nu-E_{\text {ion }} \approx 76 \mathrm{eV}$. After that, the average electron energy decreases due to inelastic collisions of electrons with the neutral background. The primary

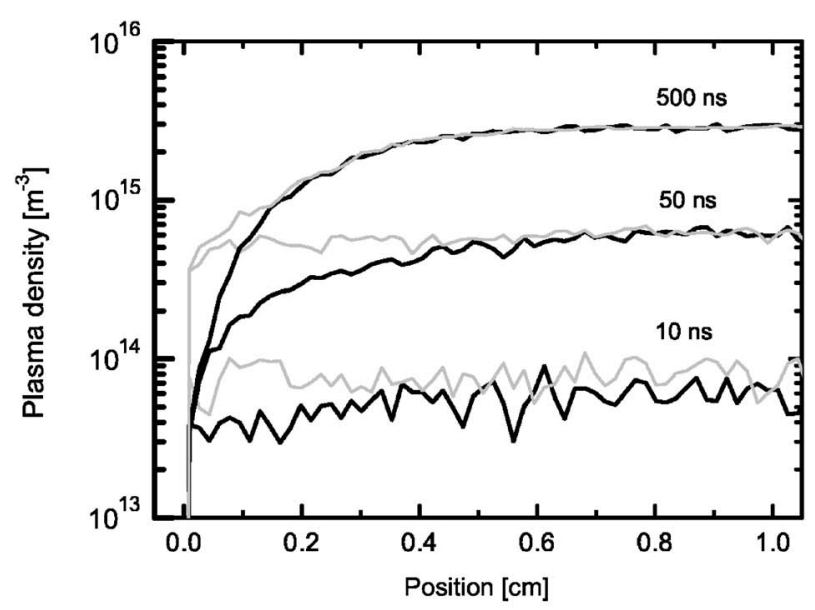

(a) No photo-effect

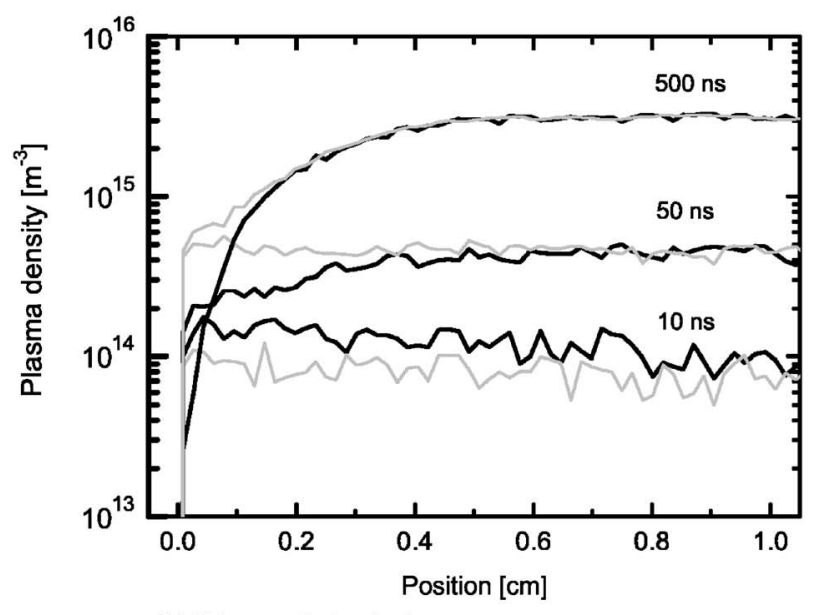

(b) Primary photo-electrons

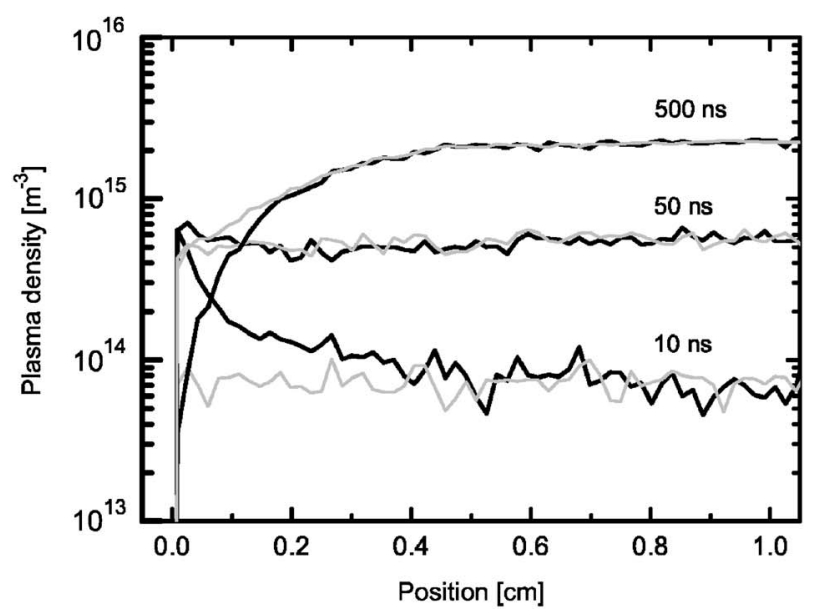

(c) Secondary photo-electrons

FIG. 4. Plasma density profile near the mirror at $t=10 \mathrm{~ns}, t=50 \mathrm{~ns}$, and $t$ $=500 \mathrm{~ns}$. The thick lines show the electron density, whereas thin lines indicate the effective ion density $n_{i}^{*}=\rho^{+} / e$. Three cases are shown: (a) no photoeffect, (b) primary photoelectrons, and (c) secondary photoelectrons.

photoelectrons slightly increase the electron energy [Fig. $5(\mathrm{~b})]$, because their initial energy $h \nu-W$ exceeds the $76 \mathrm{eV}$ at which the gas phase electrons are generated. Likewise, in Fig. 5(c), we see a strong decrease of the average electron energy, as the secondary photoelectrons have much lower energies. 


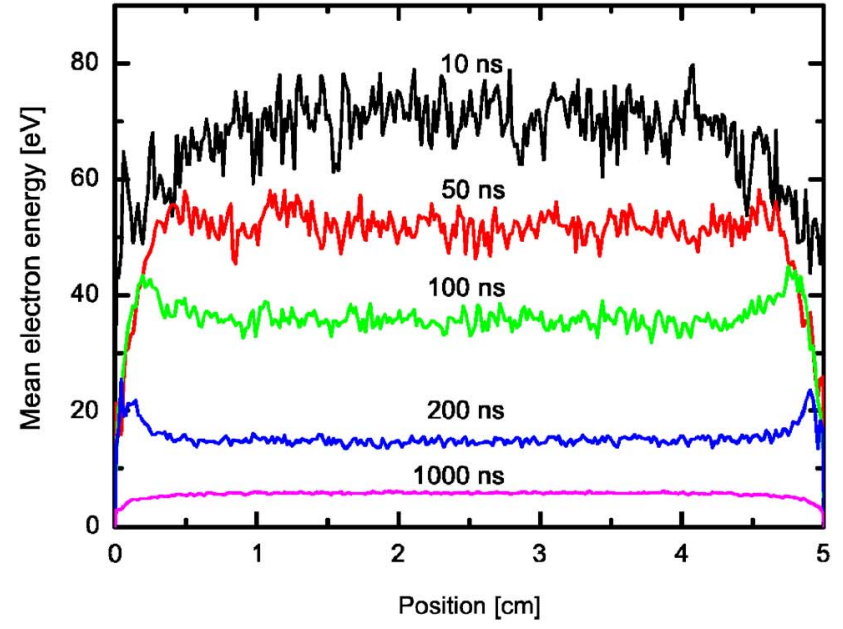

(a) No photo-effect

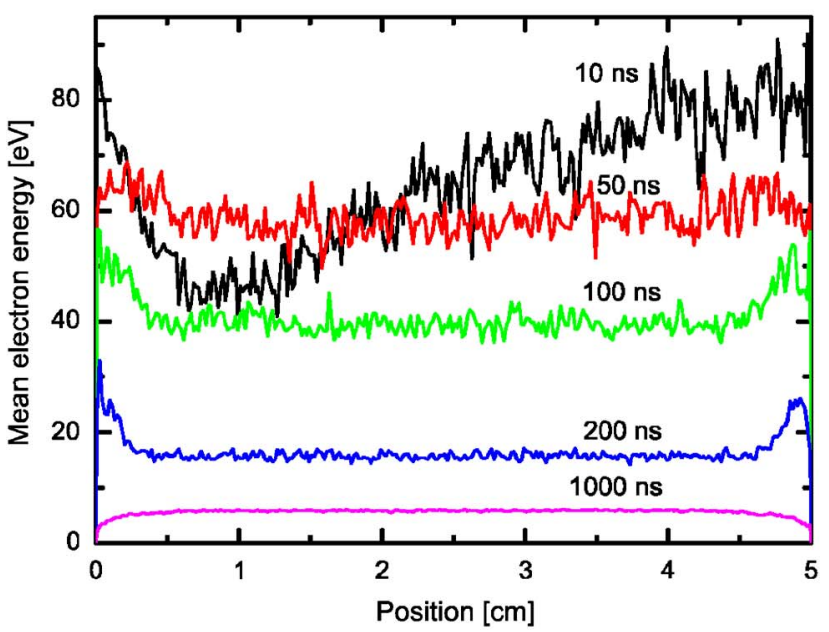

(b) Primary photo-electrons

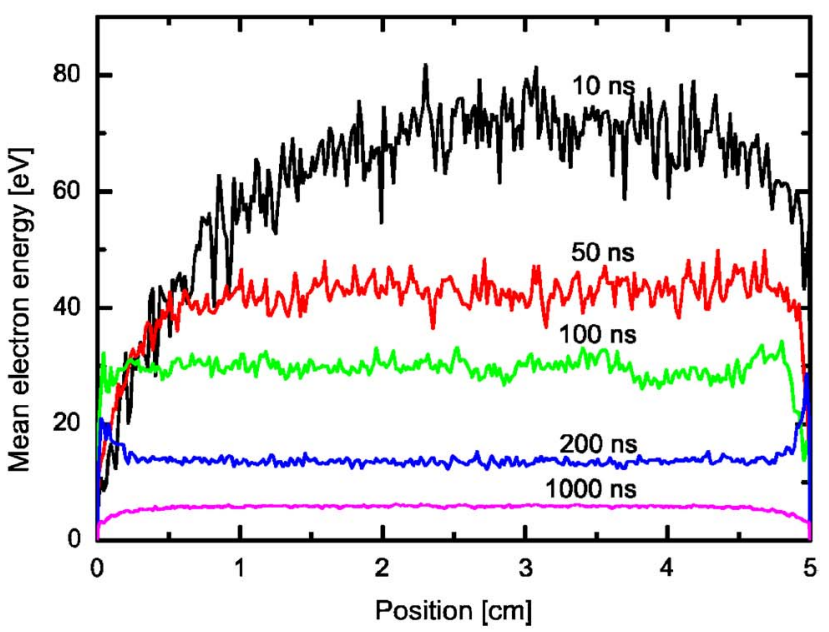

(c) Secondary photo-electrons

FIG. 5. (Color online) Cell-averaged electron energy at different times for three cases: (a) no photoeffect, (b) primary photoelectrons, and (c) secondary photoelectrons.

The formation of the plasma sheath can also be studied by considering the potential. Without photoelectric effect we obtain symmetric potential profiles, as represented in Fig. 6(a). The maximum plasma potential of $\approx 75 \mathrm{~V}$ is reached

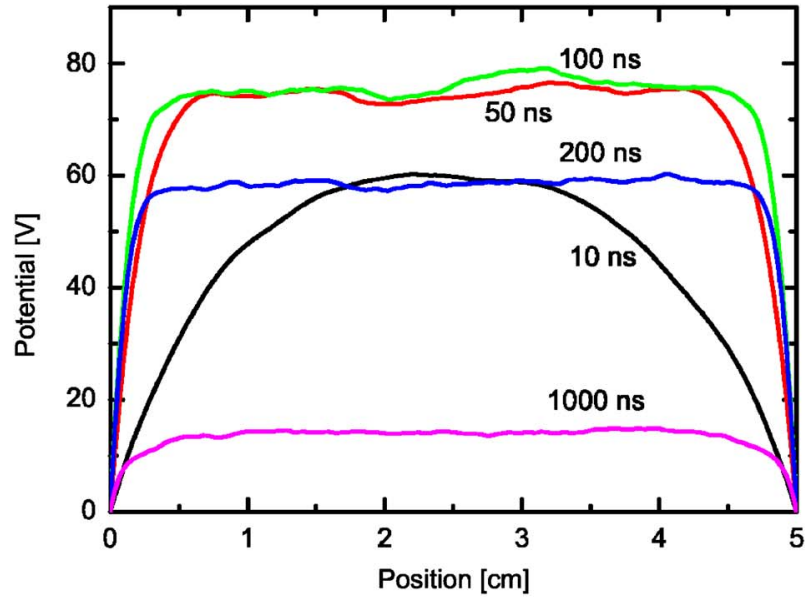

(a) No photo-effect

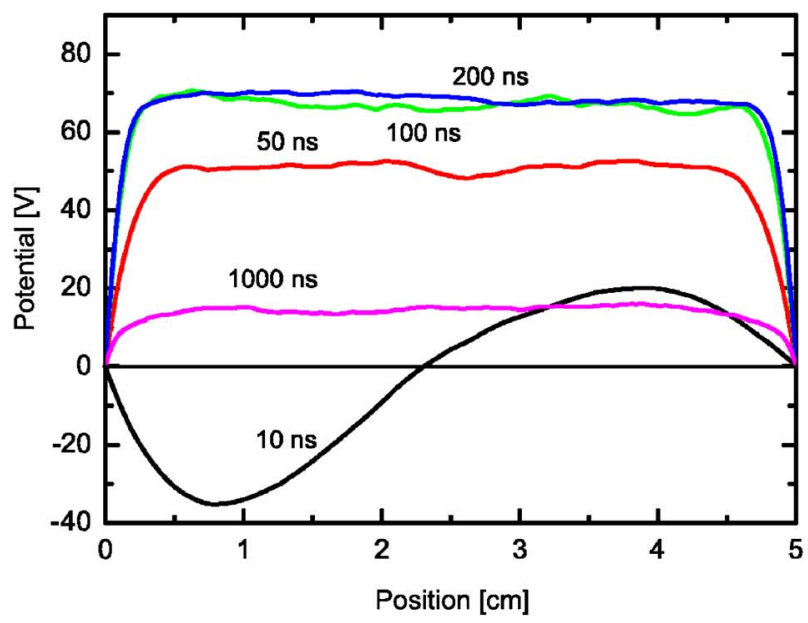

(b) Primary photo-electrons

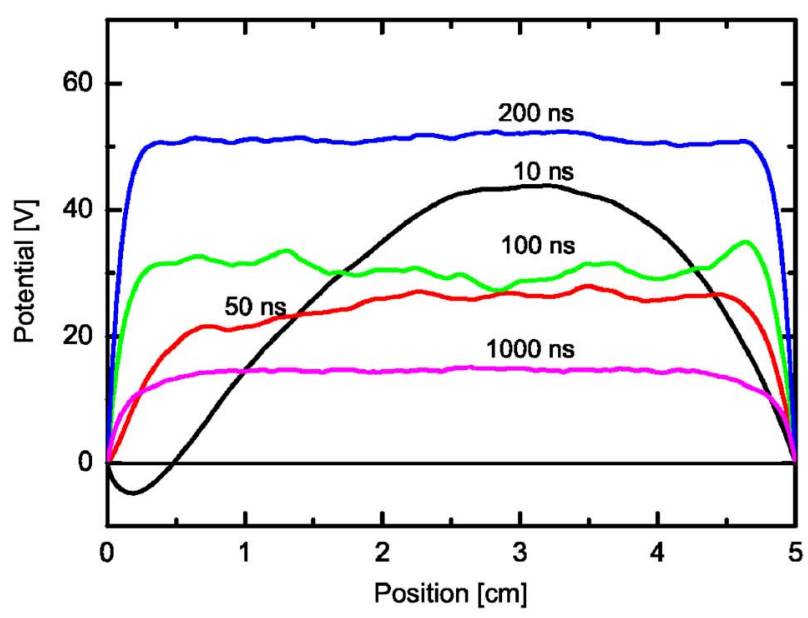

(c) Secondary photo-electrons

FIG. 6. (Color online) Potential profile at different times for three cases: (a) no photoeffect, (b) primary photoelectrons, and (c) secondary photoelectrons.

during the EUV pulse. The plasma is at a positive potential with respect to the walls as a result of the positive space charge in the sheath. After the EUV pulse $(t>100 \mathrm{~ns})$ the plasma potential gradually decreases because the average electron energy decreases. If we compare the case of no photoeffect to our earlier results, ${ }^{10}$ we find that including the 


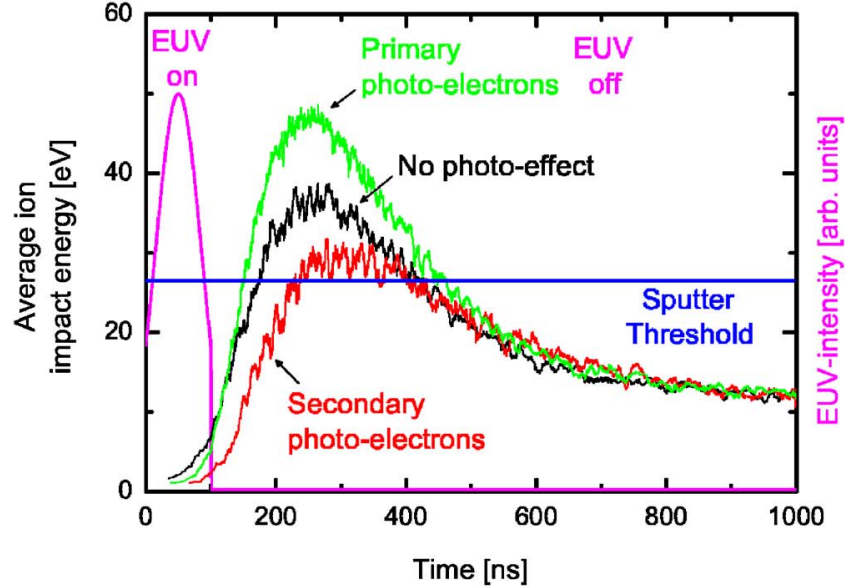

(a) $\mathrm{Ar}^{+}$ions

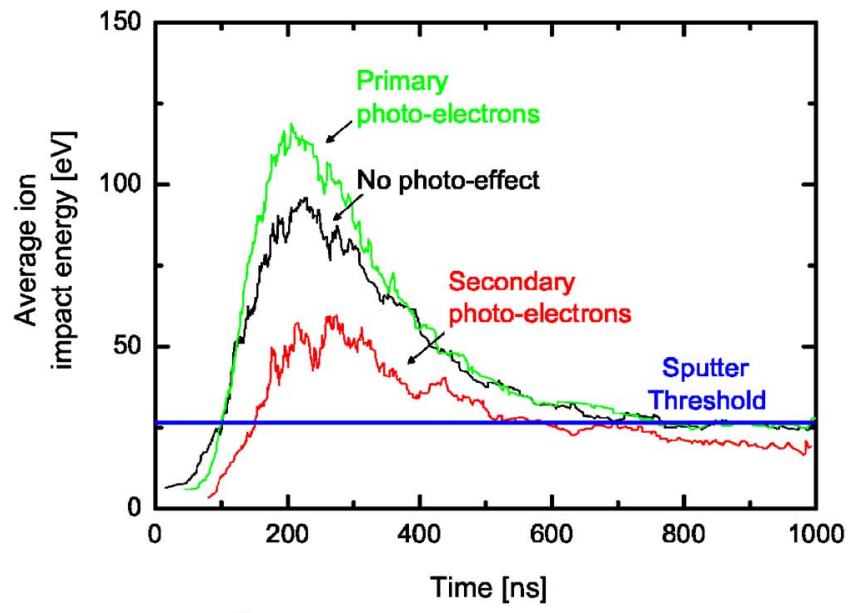

(b) $\mathrm{Ar}^{2+}$ ions

FIG. 7. (Color online) The solid lines show the energies of (a) singly and (b) doubly charged argon ions that have impacted on the mirror as a function of time. For clarity, the depicted energy is the moving average over 50 consecutive ion impacts. The horizontal line indicates the sputter threshold of Yamamura and Tawara. The dashed line in (a) shows the temporal behavior of the EUV intensity in arbitrary units.

double photoionization process to the model does not significantly alter the electron density, the mean electron energy, or the potential.

Figures 6(b) and 6(c) show the potential profiles in the case of, respectively, primary and secondary photoelectrons. The negative space charge near the mirror in the early stages of the plasma is reflected by the negative potential near the mirror at $t=10 \mathrm{~ns}$. Now, the maximum in the plasma potential occurs after the EUV pulse, because it takes more time to overcome the negative space charge and build up the positive space charge near the mirror. The maximum in the plasma potential is lower because the average electron energy is lower after the EUV pulse.

In the sheath regions, ions will be accelerated over the positive potential drop between the plasma and the walls. In Fig. 7 the energies of $\mathrm{Ar}^{+}$and $\mathrm{Ar}^{2+}$ ions impacting on the mirror are shown as a function of time. For the sake of clarity, the moving average over 50 consecutive ion impacts is taken. The dashed line represents the temporal shape of the EUV pulse. Due to their inertia the ions reach the mirror after the EUV pulse. The impact energy of $\mathrm{Ar}^{2+}$ ions is approximately two times higher compared to $\mathrm{Ar}^{+}$because, when accelerated across the same potential drop, the doubly charged ions gain twice the energy. The observation from Fig. 7 that the impact energy of $\mathrm{Ar}^{2+}$ is more than two times higher can be attributed to the fact that the $\mathrm{Ar}^{2+}$ ions reach the mirror at an earlier moment in time, when the potential drop across the sheath is still higher.

In the case of primary photoelectrons the impact energy is the highest, whereas for secondary photoelectrons it is the lowest. This is a direct consequence of the fact that the potential drop across the plasma sheath is determined by the average electron energy (Figs. 5 and 6).

\section{B. Mirror damage}

Now that the flux and energy of the ions impacting on the mirror are known, we can estimate the damage to the mirror as a result of physical sputtering. To calculate the amount of target material that is removed per EUV pulse, we convolute the ion impact energy distribution with the sputter yield $Y(E)$, i.e., the number of atoms removed from the solid per incoming ion with energy $E$. For the sputter yield the model of Yamamura and Tawara ${ }^{20}$ for light ion sputtering is used [Fig. 8(a)]. In this semiempirical model only ions with an energy above the sputter threshold contribute. The sputter threshold can be calculated from

$$
\frac{E_{\mathrm{thr}}}{U_{s}}= \begin{cases}\frac{6.7}{\lambda} & \text { for } m_{1}>m_{2}, \\ \frac{1+5.7\left(m_{1} / m_{2}\right)}{\lambda} & \text { for } m_{1} \leqslant m_{2},\end{cases}
$$

with $U_{s}$ the surface binding energy of the solid which can be approximated by the heat of sublimation ${ }^{21}$ and with

$$
\lambda=\frac{4 m_{1} m_{2}}{\left(m_{1}+m_{2}\right)^{2}}
$$

the fraction of kinetic energy that is transferred from the projectile (with mass $m_{1}$ ) to the target atom (with mass $m_{2}$ ) in case of a head-on collision. For $\mathrm{Ar}$ on $\mathrm{Ru} \lambda=0.812, U_{s}$ $=6.74 \mathrm{eV}$, and $E_{\mathrm{thr}}=27.0 \mathrm{eV}$. In Fig. 7 the sputter threshold is indicated by the horizontal line.

Figure 8 (b) shows the sputter rate in units of nanometer of mirror material removed per $10^{9}$ EUV pulses. For the case of primary photoelectrons the sputter rate is approximately two times higher compared to the case of no photoeffect and approximately seven times higher than for secondary photoelectrons. The case of no secondary photoelectrons is closest to reality, because $50 \%-90 \%$ of the emitted electrons will be low-energy secondary photoelectrons. ${ }^{19}$

The sputter rate is for the most part caused by the doubly charged ions despite the fact that the density of $\mathrm{Ar}^{2+}$ ions is more than an order of magnitude lower than the $\mathrm{Ar}^{+}$density. Sputtering by $\mathrm{Ar}^{2+}$ ions is most dominant for the case of secondary photoelectrons, because here, the impact energy of only a small fraction of the $\mathrm{Ar}^{+}$ions is above the sputter threshold. Since the ion energies are close to the sputter threshold, the calculated sputter rate is very sensitive to the 


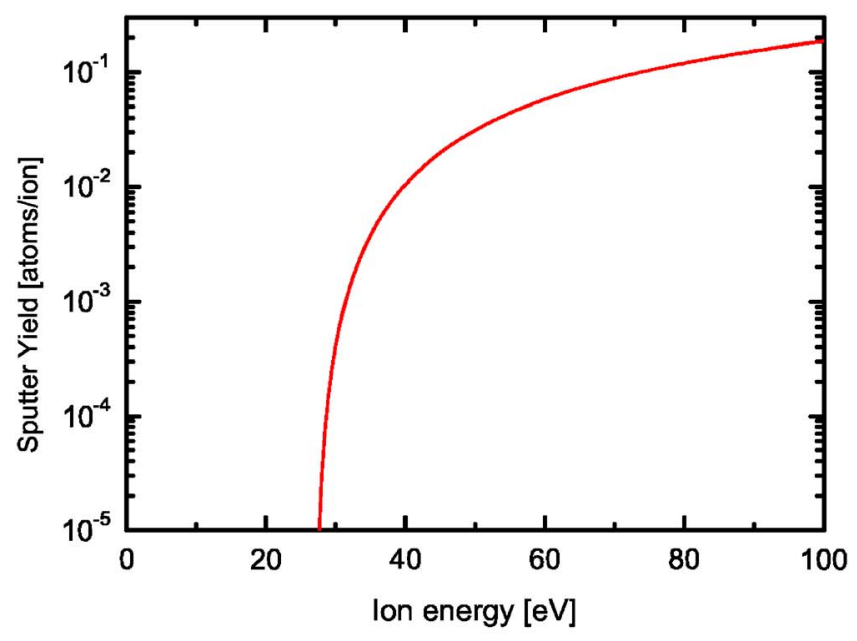

(a) Sputter yield

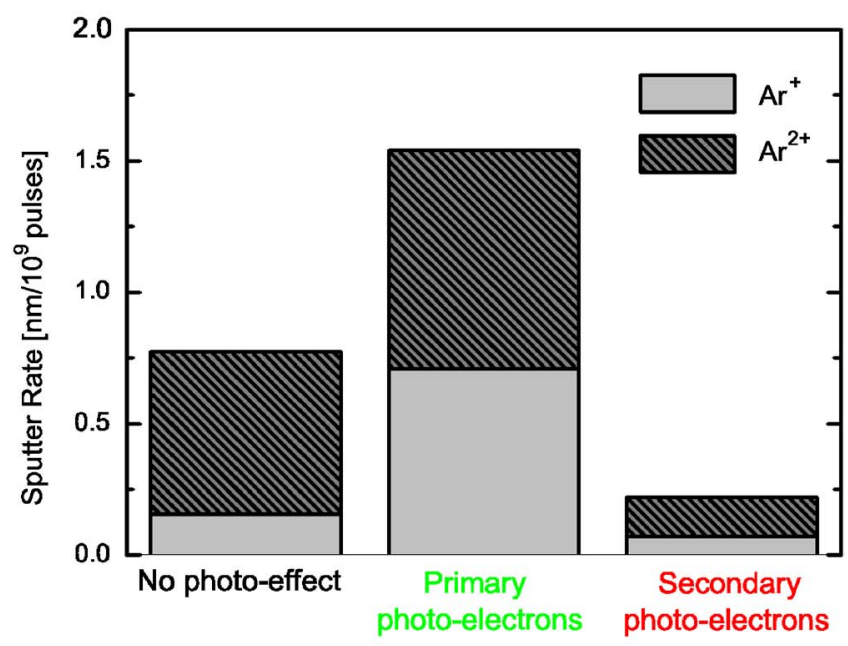

(b) Sputter rate

FIG. 8. (Color online) (a) Sputter yield as a function of ion energy for the model of Yamamura and Tawara. (b) The amount of removed mirror material in units of $\mathrm{nm} / 10^{9} \mathrm{EUV}$ pulses as a result of physical sputtering by $\mathrm{Ar}^{+}$ and $\mathrm{Ar}^{2+}$ ions for the case of no photoeffect, primary photoelectrons, and secondary photoelectrons.

value for the sputter threshold. For instance, the Bohdansky ${ }^{22}$ model for ion sputtering predicts a sputter threshold that is $10 \mathrm{eV}$ higher.

\section{CONCLUSION}

With the particle-in-cell Monte Carlo method the description of the plasma sheath region can be achieved without the need for steady-state assumptions, even for a plasma in the nonlocal regime. In addition, plasma-wall interaction processes, such as the photoelectric effect, can be incorporated.
The simulations show that only a small fraction of the ions impacting on the optical components will have sufficient energy to sputter. Therefore, the predicted sputter rate is very low $\left(\approx 1 \mathrm{~nm}\right.$ per $10^{9}$ EUV pulses $)$ and cannot be measured directly.

Photoelectrons emitted from the mirror alter the potential drop over the plasma sheath and therefore will influence the ion impact energy. The simulations predict that highly energetic, primary photoelectrons will increase the sputter rate, whereas slow, secondary photoelectrons will have the opposite effect. In reality, most of the emitted photoelectrons will be low energetic, and as a result, the photoelectric effect will reduce the sputter rate.

The sputter rate is dominated by doubly charged ions as these ions gain twice the kinetic energy of $\mathrm{Ar}^{+}$ions when accelerated over the same sheath potential drop.

\section{ACKNOWLEDGMENTS}

The authors wish to acknowledge B. Wolschrijn, D. Nijkerk, G. Alcott, and R. Moors for the valuable discussions and suggestions.

${ }^{1}$ C. W. Gwyn, R. Stulen, D. Sweeney, and D. Attwood, J. Vac. Sci. Technol. B 16, 3142 (1998).

${ }^{2}$ V. Banine, J. Benschop, M. Leenders, and R. Moors, Proc. SPIE 3997, 126 (2000).

${ }^{3}$ T. Ito and S. Okazaki, Nature (London) 406, 1027 (2000).

${ }^{4}$ E. R. Kieft, J. J. A. M. van der Mullen, G. M. W. Kroesen, and V. Banine, Phys. Rev. E 68, 056403 (2003).

${ }^{5}$ C. K. Birdsall and A. B. Langdon, Plasma Physics via Computer Simulation (Hilger, London, 1991).

${ }^{6}$ R. W. Hockney and J. W. Eastwood, Computer Simulation Using Particles (Hilger, London, 1988).

${ }^{7}$ E. Kawamura, C. K. Birdsall, and V. Vahedi, Plasma Sources Sci. Technol. 9, 413 (2000).

${ }^{8}$ C. K. Birdsall, IEEE Trans. Plasma Sci. 19, 65 (1991).

${ }^{9}$ V. Vahedi and M. Surendra, Comput. Phys. Commun. 87, 179 (1995).

${ }^{10}$ M. H. L. van der Velden, W. J. M. Brok, W. J. Goedheer, V. Banine, and J. J. A. M. van der Mullen, Phys. Rev. E 73, 036406 (2005).

${ }^{11}$ H. Gnaser, Low-Energy Irradiation of Solid Surfaces (Springer, Berlin, 1999).

${ }^{12}$ C. K. Birdsall and D. Fuss, J. Comput. Phys. 3, 494 (1969).

${ }^{13}$ H. R. Skullerud, Br. J. Appl. Phys. 1, 1567 (1968).

${ }^{14}$ A. V. Phelps and Z. L. Petrovic, Plasma Sources Sci. Technol. 8, R21 (1999).

${ }^{15}$ S. Bajt, J. B. Alameda, T. W. Barbee, Jr., W. M. Clift, J. A. Folta, B. Kaufmann, and E. A. Spiller, Opt. Eng. (Bellingham) 41, 1797 (2002).

${ }^{16}$ S. Braun, H. Mai, M. Moss, R. Scholz, and A. Leson, Jpn. J. Appl. Phys., Part 1 41, 4074 (2002).

${ }^{17}$ B. L. Henke, E. M. Gullikson, and J. C. Davis, At. Data Nucl. Data Tables 54, 181 (1993).

${ }^{18}$ S. L. Carter and H. P. Kelly, J. Phys. B 9, L565 (1976).

${ }^{19}$ B. L. Henke, J. A. Smith, and D. T. Attwood, J. Appl. Phys. 48, 1852 (1977).

${ }^{20}$ Y. Yamamura and H. Tawara, At. Data Nucl. Data Tables 62, 149 (1996).

${ }^{21}$ J. Bohdansky, J. Roth, and H. L. Bay, J. Appl. Phys. 51, 2861 (1980).

${ }^{22}$ J. Bohdansky, Nucl. Instrum. Methods Phys. Res. B 2, 587 (1984). 